\title{
Preservation of Fertility and of Reproduction Ability in Lymphoma Patients
}

\author{
Gazmend Amzai*, Aleksandar Stojanovic \\ University Clinic for Hematology, Medical Faculty, University "Sts. Cyril and Methodius", Skopje, Republic of Macedonia
}

Citation: Amzai G, Stojanovic A. Preservation of Fertility and of Reproduction Ability in Lymphoma Patients. Maced J Med Sci. 2013 June 15; 6(2): 194-201. http://dx.doi.org/10.3889/MJMS.1857-5773.2013.0292 Key words: fertility; malignant hematological disorders; lymphoma; chemotherapy; review article.

"Correspondence: Gazmend Amzai, MD. University Clinic for Hematology, Medica Faculty, University "Sts. Cyril and Methodius" Vodnjanska 17, 1109 Skopje, Republic of Macedonia.

E-Mail: dr.gazmend_amzai@hotmail.com

Received: 05-Apr-2013; Accepted: 22-Apr2013; Online first: 09-May-2013

Copyright: (๑) 2013 Amzai G. This is an openaccess article distributed under the terms of the Creative Commons Attribution License, which permits unrestricted use, distribution, and reproduction in any medium, provided the

Competing Interests: The authors have declared that no competing interests exist.

\begin{abstract}
Aim: The aim of this article is to provide the health professionals with clear, novel and practical guidelines regarding management of fertility in patients with malignant hematological disorders, with special accent on lymphoma. Also, it aims at raising consciousness of all physicians administering chemotherapy, about the undesired effects of many chemotherapy regimens, on the reproductive ability, about available methods for preserving fertility and regarding many other issues in connection with fertility in patients treated for lymphoma.
\end{abstract}

Materials and methods: Online internet databases and publications have been searched, and a systematic literature review has been performed, using the following keywords: fertility, chemotherapy as well as relevant keywords in connection to the subject.

Results: Within the search, reports regarding smaller-sized groups, as well as in series of patients and case reports have been found, but relatively few large randomized studies or actual reports regarding the success rate and the influence of methods for fertility preservation in patients treated for Hodgkin's and Non-Hodgkin's lymphoma. We have managed in summarizing a large proportion of the research studies and transferring it into an integral multidisciplinary text, offering valid and applicable options for fertility preservation in patients treated for lymphoma. We use the term lymphoma in general, since the chemotherapy and radiotherapy approaches for Hodgkin's and for Non-Hodgkin's lymphomas are similar, rendering a major part of the fertility preservation guidelines appropriate for both entities.

Recommendations: Hematologists should possess fundamental knowledge regarding the late complications of lymphoma treatment. Besides acknowledging the risk of secondary cancer development as well as of non-neoplastic cardiac and pulmonary complications, they should be prepared to raise the issue of infertility as an integral part of the treatment plan, since that is a complication of significant importance for patients treated with chemotherapy within their reproductive life period. Possible methods for preserving fertility should be presented and discussed, and patients should be referred promptly to a reproductive medicine specialist. Cryopreservation of both sperm and embryos are considered standard practice and are generally available, while other methods are still in investigational phase and performed in specialized centers under mandatory professional surveillance and expertise.

Conclusion: It is recommended to utilize treatments that are with as little as possible gonadal toxicity, to consider a wide array of options for fertility preservation as soon as possible, and to practice a decision-making process most beneficial for the patient, based on the latest medical accomplishments and most novel prospects.

\section{Introduction}

Survival rates in patients with lymphoma, as well as in all cancer patients, have greatly improved over the past 30 years. Nevertheless, the more aggressive treatment regimens, although responsible for our optimism, compromise the ability of these patients to produce biological progeny, a function of fundamental importance for their quality of life.

At present, the treatment administered in patients with Hodgkin's and Non-Hodgkin's lymphomas (all combinations of stages) can cure more than $80 \%$ of them (Diehl et al. 2003 [1]; Bernard et al. 2005 [2]), but, in spite of improving the cure rates, further diminishing of possible undesired 
treatment effects is warranted. Of particular significance are the long-term detrimental consequences, such as the risk of developing secondary infertility, as an important issue for the patients. According to a study from Cleveland, $76 \%$ of 283 young patients, who were treated for cancer and are long-term survivors, and who had no children previously, want and plan to have children, and $43 \%$ are concerned and troubled because they have never been informed of the negative treatment effects regarding reproduction [3].

A coordinated multidisciplinary joint effort is necessary, involving a hematologist, an oncologist, a team for Assisted Reproductive Technologies (ART utilization of state-of-the-art technologies for treatment of infertility), an embryologist, a researcher, a social worker and a surgeon, that will keep the patient in the center of their focus, aiming at improving the quality of patient information, assessment of treatment consequences, as well as proposals of measures for maintaining of fertility, adjusted individually for each patient. This area encompasses various medical, social and ethical aspects. Developments in this area have led to the advent of Oncofertility as an interdisciplinary link between biomedical and social sciences, studying all the issues regarding patient's fertility options, as well as choices and endpoints when assessing the diagnosis of cancer, deciding between the treatment possibilities and estimating survival prospects [4].

In order to elaborate on the gonadal toxicity of chemotherapy, we need to shortly review the normal structure and function of the gonads, known since the first descriptions of the testis, the ovary and the follicle by De Graaf (1672), and the discovery of the spermatozoid in the sperm by Leeuwenhoek (1694).

The ovaries contain large numbers of primordial follicles, reaching their maximum by the seventh month of the intrauterine development (up to 7 million). The number is reduced to between 1 and 2 million primordial follicles following birth, and decreases exponentially during life, reaching 400.000 in early puberty. During the reproductive period of a woman (30-40 years) around 1200 follicles per year suffer atresion. The ovarian reserves, i.e. the numbers of primordial follicles present at certain ages, correspond to the birth capacity and fertility of the female [5].

In contrast to this physiological (natural) condition, a pathological phenomenon can be observed, labeled premature ovarian failure (POF) and defined as an early (before the age of 40 ) loss of ovarian functions. The major consequence of this syndrome is sterility, and is characterized by amenorrhea, low estrogen levels and high gonadotropin levels. Several reasons can cause POF, but the most common is exposing the ovaries to a toxic substance, particularly to a cytotoxic agent.

Alkylating agents, most frequently implicated in the destruction of oocytes, produce covalent bonds between the DNA chains and thus prevent DNA cleavage in the process of replication, thus blocking mitosis. Such lesions cause apoptosis, not only in the proliferating cell populations (granulosa and theca cells), but in the oocytes that do not undergo divisions as well. The termination of this signaling pathway, leading to apoptosis, is presently viewed as challenging possibility for prevention of imposing damage to the ovaries by cytotoxic drugs: the inhibition of the c-Abl-TAp63 pathway, which is activated by chemotherapy in a human cell line model, as well as in murine oocytes, prevents cell death in these cells; the c-Abl kinase inhibitor blocks the proapoptotic promoter effects of cysplatinum [7]. Apart of the direct toxic effect, there are also other mechanisms explaining the pathogenesis of chemotherapy induced gonadal toxicity, such as are capillary changes with signs of fibrosis of the gonadal stromal cortex, acute vascular insult, as well oxidative stress following long-term chemotherapy.

Spermatogenesis is initiated in the testicles in early puberty and it regards a complex process with a duration of 60-70 days, through which a spermatogonium (primordial undifferentiated germ cell) develops to a mature spermatozoid. This process takes place in the seminiferous tubules and encompasses various phases of germ cell and Sertoli cell differentiation, being of essential significance for the spermatogenesis.

The possibility of spermatogenesis restoration following treatment depends on the integrity of spermatogonia and their differentiation capacity [8].

\section{Infertility risk assessment before and following treatment}

Infertility, in a sense of function, is defined as impossibility to become pregnant following a year of regular sexual intercourse without contraceptives. Fertility can be affected to a variable degree by several risk factors:

I) Individual characteristics (patient characteristics), gender, age and patient's fertility before treatment (pre-treatment fertility status of the patient),

II) Chemotherapy and radiotherapy effects attributable to the drug itself, size/location of the radiation field (whole body, brain, abdomen, bowel, etc.), dose, duration, dose-intensity, path of administration (oral vs. i.v.), as well as by

III) The disease itself - male infertility can be due to the disease itself (primary testicular lymphoma, Hodgkin's lymphoma), anatomical abnormalities (retrograde ejaculation), primary or secondary hormonal insufficiency, or, much more often, because of impairments or reduction of the germ stem cells. In women, anatomical or vascular disorders of the 
uterus, the uterine cervix or the vagina, due to the disorder itself, the operative treatment or the radiotherapy, can also prevent a naturally successful pregnancy [9].

Patient characteristics are the main risk factors for infertility. Among women treated for lymphoma, age, and with it the reserve status of the ovaries prior to the initial treatment, are of great significance for the delayed infertility risk ratio.

Within the same treatment modality, the percentage of premature ovarian failure and the subsequent resulting infertility percentage, increase with age and reach their peak after the age of 30 .

Every trial involving lymphoma in women demonstrate the influence of age on fertility.

A Norwegian study (1975-1992) analyzing 99 women (all receiving radiotherapy, and only 67 also receiving chemotherapy), showed that $37.4 \%$ of them developed Premature ovarian failure, and that women younger than 25 bear the same risk for developing POF with women over the age of 30 , but following a significantly longer time period: 15 years, as opposed to 2 years [10].

The degree of decline in the fertility capacity of women depends, as we mentioned, on the quantity of follicle reserve prior to treatment. This number can be estimated in correlation with the age as a guiding parameter, but unfortunately not with a great deal of certainty, rendering this option as not scientifically validated. On their website, Fertile Hope offers an online risk calculator (information presented and/or derived on site are not a substitute for the consult with the hemato-oncology team). In order to assess the long-term gonadal sequels following treatment, an andrological evaluation is undertaken, as well as examination of certain fertility parameters.

Many reports perceive the onset of the menstrual cycle following treatment as an important indicator of fertility. Pregnancy could not be possible without restoration of the menstrual cycle, rendering transitory amenorrhea following treatment as a risk factor for delayed infertility, and amenorrhea itself as a strong negative indicator regarding fertility. Nevertheless, according to numerous experts, only the menstrual cycle it is not a guarantee for fertility and women in whom the period is restored following chemotherapy, still carry a significant risk for an early menopause onset.

There is no certain predictor regarding the ovarian functionality following treatment, but there are many tests which can discriminate this mystery. The FSH (follicle-stimulating hormone), as an indirect indicator of the ovarian reserve, is increased in cases of ovarian dysfunction. Inhibin $B$, secreted by the granulosa cells of the follicle, is directly correlated to the degree of oocyte loss, but the assay is not very accurate.
A more reliable assay is the level of AntiMullerian hormone, a substance synthesized in the granulosa cells of the primordial follicle, having in mind that in this stage the follicles are microscopic and cannot be detected by ultrasound. With the follicle growing, synthesis decreases, and ultimately ends (no $\mathrm{AMH}$ synthesis in follicles larger than $8 \mathrm{~mm}$ ). Therefore, the blood $\mathrm{AMH}$ level reflects the ovarian reserve, and the value decreases with age (with atresion of the primordial small follicles), becoming undetectable in menopause. Women with higher $\mathrm{AMH}$ values tend to have a more favorable response to ovarian stimulation for artificial insemination [11, 12].

Many authors consider transvaginal ultrasound to be a useful ovarian reserve assay, which determines two essential elements that correlate with the reproductive reserve: assessment of ovarian volume and of the number of antral follicles $(2-8 \mathrm{~mm})$. The assay is being considered a superior one and is often used in everyday practice, does not take a lot of time and is not as expensive as the endocrinological assays [13, 14].

In contrast to women, in men treated with chemotherapy age is not an indicator of infertility, which can be explained by the fact that in men the reserves of spermatogonia are constant throughout life.

The question, an area of extensive clinical controversies, and imperative regarding lymphomas in men, is whether the gonadal dysfunction in males is primarily due to the toxic effects of chemotherapy (post-treatment infertility) or to the disorder itself (pretreatment infertility)?

Many studies, in which assessment of sperm quality was performed upon diagnosis of Hodgkin's lymphoma, revealed a high percentage of abnormal sperm specimens prior to treatment (according to some authors up to $80 \%$ ).

The results of a German study, assessing sperm quality and the hormonal status (FSH and LH) in 158 patients, with a mean age of 28 years (range: 16-52 years) are shown in Table 1 [14]. The table shows that only $30 \%$ of the patients have normal sperm parameters prior to treatment (sperm count $\geq 20 \times 10^{6} / \mathrm{mL}$, motility $\geq 50 \%$ ).

Table 1. Spermogram in patients with Hodgkin's lymphoma before treatment.

\begin{tabular}{|c|c|c|c|}
\hline Early stage & Intermediary stage & Advanced stage & $\begin{array}{l}\text { Systemic } \\
\text { symptoms }\end{array}$ \\
\hline $20(13 \%)$ & $63(40 \%)$ & $75(47 \%)$ & $67(42 \%)$ \\
\hline Azoospermia & Oligoastenospermia & $\begin{array}{l}\text { Combined } \\
\text { impairment }\end{array}$ & $\begin{array}{l}\text { Only oligo- or } \\
\text { asteno- or } \\
\text { teratospermia }\end{array}$ \\
\hline $13(8 \%)$ & 20 (13\%) & $40(26 \%)$ & $38(24 \%)$ \\
\hline \multicolumn{3}{|c|}{ Abnormal sperm: 111 (70\%) } & $\begin{array}{l}\text { Normal sperm } \\
47(30 \%)\end{array}$ \\
\hline
\end{tabular}

The results prior to treatment reveal serious impairment of fertility, as a system effect of the lymphoma itself.

According to the study, the erythrocyte 
sedimentation rate can be used as a prognostic factor of serious fertility impairment, whereas no correlation was established between the gonadotropin levels before treatment and the fertility status.

The disease stage and age have no influence on the sperm quality, whereas confirmed risk factors are the presence of general symptoms (B symptoms) at diagnosis assessment, fever, weight loss, night sweats and elevated erythrocyte sedimentation rate.

In patients with fever below $38.5^{\circ} \mathrm{C}$ only astenospermia was registered, while in patients with higher temperatures more serious sperm anomalies, including oligoastenospermia or azoospermia, were observed. The precise mechanisms are not fully understood, but involvement of proinflamatory cytokines is presumed (IL-1, IL-6, TNF- $\alpha$, soluble IL receptors 2 and 6) (Rueffer et al., 2001) [15].

The fertility status should be assessed prior to treatment in all male lymphoma patients, taking into account quantitative aspects of the sperm analysis as an essential assay, as well as hormonal markers (FSH and Inhibin B), which are also important and reveal information essential to the gonadal function.

The semen analysis, the spermogram, assesses a large number of characteristics of the semen fluid, in accordance with the World Health Organization criteria and guidelines (sperm volume, spermatozoid count, motility, morphology, shape and vitality), and, in more recent times, also the DNA integrity of the sperm (DNA fragmentation index) [16]. A high degree of DNA fragmentation correlates with the inability of the sperm to initiate fertilization, as well as with the further fate of embryonic development, if fertilization actually happens [17].

Morphological assessment of the spermatozoids is considered more subjective and difficult for comparison between different hospitals, in contrast to data obtained only regarding sperm concentration and progressive motility of the spermatozoid, which can very easily be analyzed in the lab. In order to establish a valid sperm profile, spermograms should be repeated several times following treatment (2-3 times) and not very soon after therapy, since the process of spermatogenesis are completed in 2-3 months, thus giving way to possible erroneous results.

Besides the sperm analysis, which bears an attribute of preponderance ( $90 \%$ sensitivity) and depicts the exocrine function, hormonal assays (FSH and Inhibin B) can reveal important information regarding the fertility status, in absence of the semen analysis. FSH, secreted by the adenohypophysis, increases in response to injury of the testicular tissue, due to the loss of the negative feedback mechanism [18]. Inhibin B is regarded to be a better marker than $\mathrm{FSH}$, regulating the $\mathrm{FSH}$ secretion by a mechanism of negative feedback, synthesized by the Sertoli cells, and its plasma concentration serving as the best plasma marker of spermatogenesis. Although manifesting diurnal variations closely related to testosterone, low Inhibin B values correlate well with the degree of spermatogenesis impairment [19]. Although an important spermatogenesis index, the Inhibin B assay has still only limited clinical significance in individual patient cases.

\section{Gonadal dysfunction risks related to treatment}

Gonadotoxicity of cytotoxic drugs has been known since 1948, when the first report regarding toxicity of nitrogen mustard on the testes [20].

Alkylating cytotoxic drugs (cyclophosphamide, procarbazine, melphalan, chlorambucil, busulphan) are inevitably highly associated with sterility in both men and women.

Women exposed to alkylating antineoplastic agents bear a relative infertility risk of 3.98 , compared to unexposed population, and it increases depending on the cumulative dose. The total dose of cyclophoshamide inducing ovarian failure following treatment is $20 \mathrm{gr}$ at the age of $20,9 \mathrm{gr}$ at the age of 30 and $5 \mathrm{gr}$ at the age of 40 . The incidence of gonadal dysfunction in men is not dependent on age, as in women, but correlates only with the total cyclophosphamide dose, and it is manifested in more than $80 \%$ of men post puberty, who have received more than $300 \mathrm{mg} / \mathrm{kg} \mathrm{BW}$ [21].

In Hodgkin's lymphoma, presently most often used regimens are ABVD (doxorubicine, bleomycin, vinblastine and dacarbazine) and BEACOPP (bleomycin, etoposide, doxorubicine, cyclophosphamide, vincristine, procarbazine and prednisolone), and less in use are the older regimens like MOPP, MOPPABVD, COPP and ChIVPP. Of these, only ABVD is a non-alkylating drug regimen, bearing less toxicity. The toxic effect of ABVD, as well as of the other regimens, is shown in the summary presented in table 2, where all treatment modalities and their influence on the fertility status are presented [22]. Important data are derived from the studies of the German Lymphoma Group, by Sieniawski et al. (1988-2003) [23] and the study of Behringer et al. (1994-1998) [24]. In the first study, by the German Lymphoma Group, out of 202 patients with established diagnosis of Hodgkin's lymphoma, only $20 \%$ had normospermia prior to treatment, $11 \%$ had azoospermia, and the remaining $69 \%$ had combined impairment - dysspermia.

Following therapy, only $6 \%$ of the patients had normospermia. Among these, the average time to sperm restitution was 27 months, starting at 1 year after treatment in $18 \%$, during the second year in $23 \%$, during the third year in $25 \%$, and after the third year in $35 \%$ of the patients. Patients treated with radiotherapy only, had good chances of preserving fertility, and only $11 \%$ of these developed azoospermia, in contrast to patients treated only with 
chemotherapy, who, up to $90 \%$, remained infertile, as well as those treated with the combined modality who remained azoospermic in $67 \%$ of the cases (62 of 93 patients). All patients treated with ABVD had preserved spermatogenesis following treatment (one third of them having transient azoospermia), in spite of literature data stating that the rate of permanent azoospermia in ABVD treated patients (non alkylating agent regimen) ranges from $0 \%$ to $4 \%$. The infertility rate is significantly higher in patients treated with 8xBEACOPP baseline (93\%), 8xBEACOPP escalated $(87 \%)$, as well as in patients treated with 4xCOPP/ABVD (93\%), as opposed to those receiving $2 \times C O P P / A B V D(56 \%)$. All of these regimens contain the alkylating antineoplastic drugs cyclophosphamide and procarbazine, which account for the gonadotoxicity. Only for illustration purposes, the cyclophosphamide cumulative dose after 8xBEACOPP baseline and 4xCOPP/ABVD is 5200 $\mathrm{mg} / \mathrm{sq} . \mathrm{m}$, after $8 \times B E A C O P P$ escalated it is 10.000 $\mathrm{mg} / \mathrm{sq} . \mathrm{m}$, and the cumulative dose of procarbazine is $5600 \mathrm{mg} / \mathrm{sq} . \mathrm{m}$, equivalent in all three regimens. The alkylating agents are the major risk factor for infertility, and the risk rises proportionally with the dose.

Table 2. Fertility in adult men following different treatment regimens [22].

\begin{tabular}{|c|c|}
\hline Diagnosis and treatment & Fertility following treatment \\
\hline \multicolumn{2}{|c|}{ Hodgkin's lymphoma (S. J. Howell and S. M. Shalet) [22] } \\
\hline MVPP & Azoospermia $>90 \%$ \\
\hline MOPP & Azoospermia >90\% \\
\hline ChIVPP/EVA hybrid & Azoospermia >90\% \\
\hline COPP & Azoospermia $>90 \%$ \\
\hline ABVD & $\begin{array}{l}\text { Temporary azoospermia, followed } \\
\text { by normal spermogram in almost al } \\
\text { patients after } 18 \text { months from } \\
\text { treatment completion }\end{array}$ \\
\hline \multicolumn{2}{|l|}{ non-Hodgkin's lymphoma } \\
\hline $\mathrm{CHOP}$ & Permanent azoospermia 30\% \\
\hline VAPEC-B & Normospermia $>95 \%$ \\
\hline VACOP-B & Normospermia $>95 \%$ \\
\hline MACOP-B & Normospermia $>95 \%$ \\
\hline VEEP & Normospermia $>95 \%$ \\
\hline \multicolumn{2}{|l|}{ Bone marrow transplantation } \\
\hline Cyclophosphamide only & FSH increases in $>40 \%$ \\
\hline Busulphan and cyclophosphamide & FSH increases in $>80 \%$ \\
\hline CBV & FSH increases in $>95 \%$ \\
\hline High-dose melphalan & FSH increases in $>95 \%$ \\
\hline BEAM & $\mathrm{FSH}$ increases in $>95 \%$ \\
\hline \multicolumn{2}{|l|}{ Testicular carcinoma } \\
\hline Cysplatinum/carboplatinum based therapies & $\begin{array}{l}\text { Normospermia in } 50 \% \text { within } \\
\text { years and in } 80 \% \text { within } 5 \text { years }\end{array}$ \\
\hline \multicolumn{2}{|c|}{ Hodgkin's lymphoma (Sieniawski et al. 2008 - 112 pts) [23] } \\
\hline ABVD & Azoospermia 0\% \\
\hline 2 COPP/ABVD & Azoospermia 56\% \\
\hline 4 COPP/ABVD & Azoospermia 91\% \\
\hline 8 BEACOPP baseline & Azoospermia 93\% \\
\hline 8 BEACOPP escalated & Azoospermia 87\% \\
\hline Radiotherapy & Azoospermia 11\% \\
\hline
\end{tabular}

MVPP = mustine, vinblastine, procarbazine and prednisolone; MOPP = mustine vincristine, procarbazine and prednisolone; ChIVPP/EVA = chlorambucil, vinblastine, prednisolone, procarbazine, doxorubicin, vincristine and etoposide; COPP = cyclophosphamide, vincristine, procarbazine and prednisolone; $A B V D=$ doxorubicin hydrochloride, bleomycin, vinblastine and dacarbazine; $\mathrm{CHOP}=$ cyclophosphamide, doxorubicin, vincristine and prednisolone; VAPEC-B = vincristine, doxorubicin, prednisolone, etoposide, cyclophosphamide and bleomycin; VACOP-B = vinblastine, doxorubicin, prednisolone,vincristine, cyclophosphamide and bleomycin; MACOP-B = mustine in place of vinblastine VEEP $=$ vincristine, etoposide, epirubicin and prednisolone $\mathrm{CBV}=$ cyclophosphamide, carmustine and etoposide; $\mathrm{BEAM}=$ carmustine, etoposide Ara-C and melphalan; BEACOPP (bleomycin, etoposide, doxorubicin, cyclophosphamide, vincristine, procarbazine, prednisolone); $\mathrm{FSH}=$ follicle-stimulating hormone.

In the study of Behringer et al. [24] the object of analysis was secondary amenorrhea following treatment in 405 women with Hodgkin's lymphoma, following a mean observation period of 3.2 years. The highest risk rate for amenorrhea, considering all presently used regimens for treatment of Hodgkin's lymphoma, is conveyed by regimens containing high doses of alkylating agents (ABVD 3.9\%, baseline BEACOPP 22.6\%, escalated BEACOPP 51.4\%), women with advanced stage disease and those older than 30. The conclusions of the German Hodgkin's Lymphoma Study Group in this trial suggest that compromising fertility is largely due to the influence of the type and extent of chemotherapy (chemotherapy regimen), age, disease stage, and, somewhat surprising, to the utilization of oral contraceptives during treatment with the aim of ovarian impairment prevention. This information is available in the literature, but many researchers have not confirmed any protective effect whatsoever, in women receiving oral contraceptives simultaneously with the chemotherapy [25].

It is evident that both in men and in women the incidences, as well as the risk for fertility, increases when alkylating agents are being used. Decanter et al. compared the serum levels of AntiMullerian hormone $(\mathrm{AMH})$ in patients treated with $A B V D$ and with non-ABVD regimens (regimes containing cyclophosphamide). It was already stated that $\mathrm{AMH}$ serves as a parameter of evolution in the process of follicular depletion of the ovaries, in patients treated for lymphoma. The AMH assays were performed prior to, and during chemotherapy, and every three months following treatment cessation, during a one-year period. In all patients, the $\mathrm{AMH}$ concentration declined drastically immediately following the onset of chemotherapy, and remained very close to the detection limit level until the end of treatment. In the ABVD treated group, the $A M H$ concentration started to rise from the third month after therapy cessation and reached the levels before treatment in 12 months from the end of therapy. In contrast, no significant changes have been noted in the non-ABVD treated group, and the $A M H$ levels had remained undetectable during the follow-up period [26].

In patients with non-Hodgkin's lymphomas, who most frequently receive $\mathrm{CHOP}$ or CHOP-like regimens, the observed rate of gonadal toxicity, in both men and women, has been quite low [27]. An Israeli study, performed on 36 women under the age of 40 having aggressive non-Hodgkin's lymphoma in first remission following treatment, fertility and pregnancy rates before therapy had been analyzed, as well as during and after treatment [28]. All patients had received chemotherapy (CHOP) and only 10 had received additional radiotherapy. At the moment of diagnosis assessment $86 \%$ of the women had a regular period, and during therapy $50 \%$ developed amenorrhea, $17 \%$ experienced irregularities in the period, and $33 \%$ went on without changes in the period regularity. Following treatment, the menstrual cycle had been restored in all patients (in $63 \%$ within 3 months after chemotherapy completion, and 50\% 
had also become pregnant during first complete remission), except in two women who remained amenorrheic, who were at the age of 40 at diagnosis assessment. In conclusion, it can be said that gonadal dysfunction is insignificant in young female patients with non-Hodgkin's lymphoma treated with $\mathrm{CHOP}$ chemotherapy, rendering contemporary methods for preserving fertility unnecessary in such cases of female patients under the age of 40 .

Gonadal toxicity of second-line treatment, used in relapsed and refractory lymphomas, such as DHAP (dexamethasone, high-dose cytarabine, cysplatinum), MINE (mesna, iphosphamide, mitoxantrone, etoposide), ICE (iphosphamide, carboplatinum, cysplatinum) and GVD (gemcitabine, vinorelbine, doxorubicine liposomal), remain undetermined.

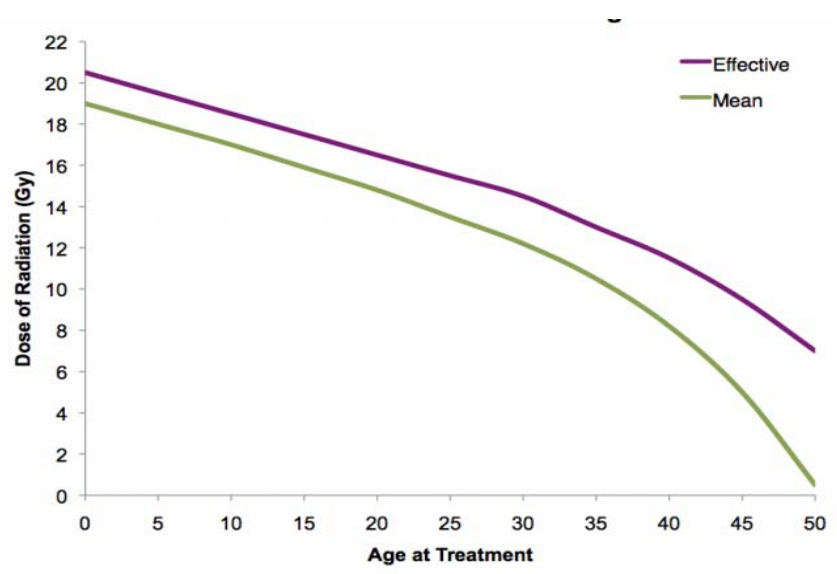

Figure 1: Effective and mean sterilization dose, from birth to the age of 50 [29]. Effective dose - upper curve (radiation dose that would reduce the number of primordial follicles below $\sim 1000$ and would cause permanent ovarian failure in $97.5 \%$ of the women); Mean sterilization dose - lower curve (radiation dose that would cause permanent ovarian failure in $50 \%$ of the women).

The radiation risk for fertility depends on the field size and of the therapeutic dose administered. The radiosucceptibility of the gonads has been well documented. In 2005, Wallace et al. had calculated the viable follicles following a different administered radiation dose, by means of which the radiosensitivity of the human oocyte has been estimated: $L_{50}$ (the dose that would kill $50 \%$ of the cells) is lower than 2 Gy. A radiation dose of 2.5 - $5.0 \mathrm{~Gy}$, would render $30 \%$ of the women between the ages of 15 and 40 , and $>90 \%$ of the women above the age of 40 , infertile. The dose of effective sterilization, causing premature ovarian failure in more than $97.5 \%$ of women, decreases with advanced age. Immediately after birth it is $20.3 \mathrm{~Gy}$, at the age of 10 it is $18.4 \mathrm{~Gy}$, at the age of 20 it is $16.5 \mathrm{~Gy}$, and at the age of 30 it is $14.3 \mathrm{~Gy}$ (Figure 1) [29]. A radiation dose of more than $21 \mathrm{~Gy}$ induces permanent ovary ablation, while a smaller dose would induce changes dependent on the patient's age, thus noting functional ovary changes even following a dose of $1.5 \mathrm{~Gy}$ in patients over 40 .

The testicles are among the most radiosensitive organs, and even low dose irradiation can significantly impair their function. Reduction in spermatozoid count is observed following a dose of $0.15 \mathrm{~Gy}$, and risk for sterilization following 1-2 Gy. For them, the rule that the more immature, the more radiosensitive the cell is, is applicable, therefore allowing morphological and quantitative changes in spermatogonia to be observed even with doses lower than 0.1 Gy [30, 31]. Radiotherapy over the abdominal and pelvic regions, as part of the treatment plan, has severe detrimental effects on gonadal function, rendering radiotherapy below the diaphragm as a first choice therapy obsolete, and classifying indications for such use rare and exceptional. Following supradiaphragmal radiotherapy, the dose to the gonads is around $0.1 \%$, showing that adaptive radiotherapy, under strict three-dimensional control of the radiation field (imaging adapted) enables more precise confinement of the targeted regions, whereas the modern era of computer directed technology adjusts the radiation dose, considering both the tumor and safeguarding the normal healthy tissue (knowing the tolerance dose of normal tissues).

Pelvic irradiation can also cause fibrosis of the uterus, which in turn can compromise future pregnancy. Cranial irradiation with a dose higher than 35 Gy can damage the hypothalamic-hypophiseal axis and cause a hypogonadal condition and infertility due to secondary hormonal insufficiency.

\section{Various methods for fertility preservation}

The ability to preserve fertility, to conserve gametes, embryos or gonadal tissue by various methods, has become one of the cardinal issues for lymphoma survivor patients treated with radio- and chemotherapy. Several options are available and should be adequate for defined patient categories, depending on patient's age, existence or absence of a partner and patient's desires. The use of some available techniques is still controversial, with undetermined success rates, based only on a few case reports, expert opinions and small-sized retrospective analyses, which is why they should be considered experimental.

Embryo cryopreservation is the most successful available method up to date, being performed routinely in many centers, in many infertile patients, producing reputable results. The procedure involves hyperstimulation of the ovaries and the induction of multifollicular growth, since the collection of only one ovum, maturing in a natural menstrual cycle, has a lesser than $10 \%$ chance to be fertilized, survive the freezing and result in a viable fetus following the migration to the uterus. The mature oocytes are collected and are fertilized in vitro (IVF) with the partner's sperm, and the resulting embryos are being cryopreserved. In order to secure appropriate viability of the embryo, well defined freezing and defreezing (thawing) protocols for human 
embryos are implemented. At the proper point of time, the embryo is implanted. The rate of successful pregnancies after every single implanted embryo is $18 \%(8-30 \%)$ and the cumulative rate of pregnancies for the entire procedure is $>60 \%$. The limitations of this technique are that it is applicable only in women post puberty, within their regenerative period, with a committed partner, the supraphysiological levels of estrogen used during ovarian stimulation as well as the delay in treatment, issues that need to be discussed respecting the disease stage and the desires and plans of the patient, since the time-frame from the ovarian stimulation to the cryopreservation of the embryo can take even several weeks. Interested parties should be informed that the cost of procedure amounts to $\$ 5000$ - $\$ 8000$ per cycle, with additional annual $\$ 350$ for storage expenses (thus becoming a negative criterion in countries with a lower index of economic development) [31-35].

Sperm cryopreservation is a much easier, more efficient and more effective method for fertility preservation in adult men and in adolescents post puberty. Several techniques for sperm collection are in use, like ejaculated sperm obtained by masturbation, rectal electroejaculation, etc. [36]. In cases of azoospermia, sperm can be obtained by testicular aspiration or testicular biopsy, in about half of the cases. The obtained sperm specimen is analyzed, and then diluted with a cryoprotectant, frozen by liquid nitrogen vapor and then placed in storage reservoirs for extended conservation in liquid nitrogen. Averages of 2-4 collections are needed, each following a 48-hour abstinence period [37].

In many patients, and if the sperm quality is poor, or in oligospermic subjects, the ejaculate can still be used with assisted reproductive technologies, such as intrauterine insemination, simple in vitro fertilization or in vitro fertilization with intracytoplasmic sperm injection (ICSI)[31].

Aside from these two, established and standardized methods for fertility preservation, there are also other potential options, currently under investigation, but producing encouraging results. In women, it is possible to cryopreserve mature oocytes, to cryopreserve ovarian tissue and to suppress the ovaries with gonadotropin releasing hormone $(\mathrm{GnRH})$ analogues. Several techniques have been under investigation, still remaining experimental, in boys pre puberty: cryopreservation of immature testicular tissue and cryopreservation of stem germ cells, which would eventually be reimplanted in the testes [9, 38-40].

\section{Conclusions}

The diagnosis and treatment of a malignant disease often represents a threat to fertility, whereas studies show that the biological ability to have children is of great importance for a lot of people. Therefore, a whole array of options needs to be planned as early as possible, so that fertility is preserved.

Decisions should be made that are best for the patient and based on the latest medical achievements and on most novel prospects.

The sperm of patients with $\mathrm{HL}$ and $\mathrm{NHL}$ prior to treatment is of lower quality, compared to the healthy male control population.

Gonadal toxicity is minimal in patients with non-Hodgkin's lymphoma treated with $\mathrm{CHOP}$ chemotherapy. In male patients with Hodgkin's lymphoma sperm should be collected before BEACOPP chemotherapy, as well as prior to salvage chemotherapy in relapsed patients, whereas regarding patients treated with the ABVD regimen a valid option remains that they should reach their own decision (following an expert consultative discussion with a hematologist), considering the low risk rate of infertility following ABVD chemotherapy (in most patients normal spermatogenesis is restored within 618 months following ABVD chemotherapy).

In women there are no recommendations for using any method of preserving fertility if they are going to receive ABVD chemotherapy, but such can be recommended in patients older than 35 , and that decision is made according to patient's desire. For women treated with BEACOPP chemotherapy an expert consult with a reproduction specialist should be offered, and the decision is made individually and personally, considering the expert opinion.

\section{Acknowledgements}

Publication fee for this review article was covered by Ss Cyril and Methodius University Skopje PhD School.

\section{References}

1. Diehl V, Franklin J, Pfreundschuh $M$, Lathan B, Paulus U, Hasenclever D, Tesch H,Herrmann R, Dorken B, Muller-Hermelink $\mathrm{HK}$, et al. Standard and increased-dose BEACOPP chemotherapy compared with COPP-ABVD for advanced Hodgkin's disease. N Engl J Med. 2003; 348(24):2386-95.

2. Bernard M, Cartron G, Rachieru P, LeMevel A, Branger B, Le Maignan C, Berthou C, Ghandour C,Delwail V, Milpied N, et al. Long-term outcome of localized high-grade non-Hodgkin's lymphoma treated with high dose $\mathrm{CHOP}$ regimen and involved field radiotherapy: results of a GOELAMS study. Haematologica. 2005; 90:802-809.

3. Schover LR, Rybicki LA, Martin BA, Bringelsen KA. Having children after cancer: A pilot survey of survivors' attitudes and experiences. Cancer. 1999;86:697-709.

4. Chevalier N, Dewailly D, Fenichel P. Oncofertility: a new focus in women health-care. Ann Endocrinol (Paris). 2009;70(Suppl 1):S3341.

5. Faddy MJ, Gosden RG. A mathematical model of follicle dynamics in the human ovary. Hum Reprod. 1995;10:770-775.

6. Shelling AN. Premature ovarian failure. Reproduction. 2010; 140(5):633-641. 
7. Gonfloni S, Di Tella L, Caldarola S, Cannata SM, Klinger FG, Di Bartolomeo C, et al. Inhibition of the C-Abl-TAp63 pathway protects mouse oocytes from chemotherapy-induced death. Nat Med. 2009;15(10):1179-85.

8. Heller, C.G.; Clermont, Y. (April 1963). "Spermatogenesis in Man: An Estimate of Its Duration". Harel S, Fermé C, Poirot C. Management of fertility in patients treated for Hodgkin's lymphoma. Haematologica. 2011;96(11):1692-9.

9. Lee SJ, Schover LR, Partridge AH, Patrizio P, Wallace $W H$, Hagerty K, Beck LN, Brennan LV, Oktay K. American Society of Clinical Oncology: American Society of Clinical Oncology recommendations on fertility preservation in cancer patients. J Clin Oncol. 2006; 24: 2917-2931.

10. Haukvik UK, Dieset I, Bjøro T, Holte H, Fosså SD. Treatmentrelated premature ovarian failure as a long-term complication after Hodgkin's lymphoma. Ann Oncol. 2006;17(9):1428-33.

11. Jenny A Visser, Frank $H$ de Jong, Joop S E Laven I and Axel PN Themmen. Anti-Müllerian hormone: a new marker for ovarian function. Reprodution. 2006;131:1-9.

12. van Beek RD, van den Heuvel-Eibrink MM, Laven JSE, de Jong FH, Themmen APN, Hakvoort-Cammel FG, et al. Anti-Mullerian hormone is a sensitive serum marker for gonadal function in women treated for Hodgkin's lymphoma during childhood. J Clin Endocrinol Metab. 2007;92(10):3869-74.

13. Broekmans FJ, Faddy MJ, Scheffer G, te Velde ER. Antral follicle counts are related to age at natural fertility loss and age at menopause. Menopause. 2004; 11(6 Pt 1):607-14.

14. Kwee J, Elting ME, Schats R, McDonnell J, Lambalk CB Ovarian volume and antral follicle count for the prediction of low and hyper responders with in vitro fertilization. Reprod Biol Endocrinol. 2007;5:9.

15. Rueffer U, Breuer K, Josting A, Lathan B, Sieber M, Manzke O, Grotenhermen FJ, Tesch H, Bredenfeld H, Koch P, Nisters-Backes $\mathrm{H}$, Wolf $\mathrm{J}$, Engert A, Diehl V. Male gonadal dysfunction in patients with Hodgkin's disease prior to treatment. Ann Oncol. 2001;12(9):1307-11.

16. Smit M, van Casteren NJ, Wildhagen MF, Romijn JC, Dohle GR. Sperm DNA integrity in cancer patients before and after cytotoxic treatment. Hum Reprod. 2010;25(8):1877-83.

17. Muriel L, Garrido N, Fernández JL, Remohí J, Pellicer A, de los Santos MJ, Meseguer M.Value of the sperm deoxyribonucleic acid fragmentation level, as measured by the sperm chromatin dispersion test, in the outcome of in vitro fertilization and intracytoplasmic sperm injection. Fertil Steril. 2006; 85(2):371-83.

18. van der Kaaij MA, Heutte N, Le Stang N, Raemaekers JM, Simons AH, Carde P, et al. Gonadal Function in Males Afte Chemotherapy for Early-Stage Hodgkin's Lymphoma Treated in Four Subsequent Trials by the European Organisation for Research and Treatment of Cancer: EORTC Lymphoma Group and the Groupe d'Etude des Lymphomes de l'Adulte. J Clin Oncol. 2007; 25:2825-32

19. van Beek RD, Smit M, van den Heuvel-Eibrink MM, de Jong FH Hakvoort-Cammel FG, van den Bos C, van den Berg H, Weber RF, Pieters R, de Muinck Keizer-Schrama SM. Inhibin B is superior to $\mathrm{FSH}$ as a serum marker for spermatogenesis in men treated for Hodgkin's lymphoma with chemotherapy during childhood. Hum Reprod. 2007; 22: 3215-3222.

20. Spitz S. The histological effects of nitrogen mustards on human tumors and tissues. Cancer. 1948;1(3):383-98.

21. Rivkees SA, Crawford JD. The relationship of gonadal activity and chemotherapy-induced gonadal damage. JAMA. 1988;259:2123 - 5 .

22. Howell SJ, Shalet SM. Spermatogenesis after cancer treatment: damage and recovery. J Natl Cancer Inst Monogr. 2005;(34):12-7.

23. Sieniawski M, Reineke $T$, Josting A, Nogova L, Behringer $K$, Halbsguth $T$, et al. Assessment of male fertility in patients with Hodgkin's lymphoma treated in the German Hodgkin Study Group
(GHSG) clinical trials. Ann Oncol. 2008;19(10):1795-801.

24. Behringer K, Breuer K, Reineke T, May M, Nogova L, Klimm B, et al. Secondary amenorrhea after Hodgkin's lymphoma is influenced by age at treatment, stage of disease, chemotherapy regimen, and the use of oral contraceptives during therapy: a report from the German Hodgkin's Lymphoma Study Group. J Clin Oncol. 2005;23(30):7555-64

25. Whitehead E, Shalet SM, Blackledge G, et al: The effect of combination chemotherapy on ovarian function in women treated for Hodgkin's disease. Cancer. 1983; 52(6):988-93.

26. Decanter C, Morschhauser F, Pigny P, Lefebvre C, Gallo C, Dewailly D. Anti-Müllerian hormone follow-up in young women treated by chemotherapy for lymphoma: preliminary results. Reprod Biomed Online. 2010; 20(2):280-5.

27. Dann EJ, Epelbaum R, Avivi I et al. Fertility and ovarian function are preserved in women treated with an intensified regimen of cyclophosphamide, adriamycin, vincristine and prednisone (MegaCHOP) for non-Hodgkin lymphoma. Hum Reprod. 2005; 20(8):2247-9.

28. Elis A, Tevet A, Yerushalmi R, Blickstein D, Bairy O, Dann EJ, Blumenfeld Z, Abraham A, Manor Y, Shpilberg O, Lishner M. Fertility status among women treated for aggressive non-Hodgkin's lymphoma. Leuk Lymphoma. 2006;47(4):623-7.

29. Wallace WHB, Thomson AB, Seran F,Kelsey TW. Predicting ovarian failure after radiation to a field that includes the ovaries.Int $\mathrm{J}$ Radiation On clogy Biol Phys. 2005;62(3):738-44.

30. Rowley MJ, Leach DR, Warner GA, Heller CG. Effect of graded doses of ionizing radiation on the human testis. Radiat Res. 1974; 59(3):665-78.

31. Harel S, Fermé C, Poirot C. Management of fertility in patients treated for Hodgkin's lymphoma. Haematologica. 2011;96(11): 1692-1699.

32. Al-Shawaf $\mathrm{T}$, Yang $\mathrm{D}$, al-Magid $\mathrm{Y}$, Seaton $\mathrm{A}$, Iketubosin $\mathrm{F}$ and Craft I (1993) Ultrasonic monitoring during replacement of frozen/thawed embryos in natural and hormone replacement cycles. Hum Reprod. 1993;8(12):2068-74.

33. Wang JX, Yap YY and Matthews CD (2001) Frozen-thawed embryo transfer: influence of clinical factors on implantation rate and risk of multiple conception. Hum Reprod. 2001;16(11):2316-9.

34. Frederick JL, Ord T, Kettel LM, Stone SC, Balmaceda JP and Asch RH (1995) Successful pregnancy outcome after cryopreservation of all fresh embryos with subsequent transfer into an unstimulated cycle. Fertil Steril. 1995;64 (5): 987-90.

35. Selick CE, Hofmann GE, Albano C, Horowitz GM, Copperman $A B$, Garrisi GJ and Navot D. Embryo quality and pregnancy potential of fresh compared with frozen embryosĐis freezing detrimental to high quality embryos? Hum Reprod. 1995;10:392395.

36. Bahadur G, Ling KL, Hart R, Ralph D, Riley V, Wafa R, Ashraf A, Jaman N, Oyede AW. Semen production in adolescent cancer patients. Hum Reprod. 2002;17(10):2654-6.

37. Sanger WG, Olson JH, Sherman JK. Semen cryobanking for men with cancer criteria change. Fertil Steril. 1992; 58(5):1024-7.

38. Avarbock MR, Brinster CJ, Brinster RL. Reconstitution of spermatogenesis from frozen spermatogonial stem cells. Nat Med. 1996; 2(6):693-6.

39. Brinster RL. Germline stem cell transplantation and transgenesis. Science. 2002;296(5576):2174-6.

40. Brook PF, Radford JA, Shalet SM, Joyce AD, Gosden RG.Isolation of germ cells from human testicular tissue for low temperature storage and autotransplantation. Fertil Steril. 2001; 75 (2):269-74 